\title{
Experimental Studies on Permeability of the Pulmonary Capillary
}

\author{
Tatsuya Tomomatsu, M.D., Tsutomu Inoh, M.D., Yutaka \\ Yoshida, M.D., and Kiyoyasu OKa, M.D.
}

HE rate of passage of substance across the capillary wall measured in
terms of increased quantity (ds) passing through during a period of time $(\mathrm{dt})$ is directly proportional to the area $(\mathrm{A})$ of the wall, the driving force between the inside $(\mathrm{Gi})$ and the outside $(\mathrm{Co})$ of the capillaries, and the diffusion constant $(\mathrm{K})$ for the substance, and is inversely proportional to the thickness $(\theta)$.

$$
\mathrm{ds} / \mathrm{dt}=\mathrm{A} / \theta(\mathrm{Ci}-\mathrm{Co})^{1)}
$$

The area of the wall, namely, the area of the pulmonary capillary wall depends on the venous return and pulmonary blood volume, and the driving force is the pressure gradient between the hydrostatic pressure of the pulmonary capillary and the tissue pressure of the lung. These 2 components are variable with the hemodynamic changes.

The thickness of the capillary wall is related to its diameter, depending on the capillary blood volume. So it may be variable not only by the hemodynamic change, but also by the injury due to chemicals, ${ }^{2-7)}$ gases and some other physical factors. ${ }^{8,9}$,

The hemodynamic changes related to an increased permeability, such as the elevated pulmonary capillary pressure and increased pulmonary capillary blood volume, may be caused by an increased vasomotor activity clicited by stimulation of the central nervous system by various means..$^{10)-13)}$

An increased capillary filtrate is drawn back by the effective osmotic pressure to the capillary distal to the venule to prevent from accumulation of the excessive extracellular fluic.

Some part will be drained through the lymphatic channels to the superior vena cava.

If the pulmonary transudation is increased beyond an attractive force of the effective osmotic pressure to drawn fluid inward, then the lymph flow is bound to be increased.

Lowering of the osmotic pressure as well as obstruction of the lymphatic Kobe.

From the Department of Internal Medicine, Division I, Kobe University School of Medicine, 
channels would provide auxiliary factors to retain interstitial fluid.

So far as the lymphatic flow is found to be increased concomitantly with enhanced transudation of the pulmonary capillary, it is reasonably assumed that measurement of the lymph flow can be served as an index of the permeability of the lung capillary.

In this report, attempts have been made to measure the lymph flow of the lung to know the effect of the sympathomimetic drug and anoxia both of which are considered to offer a number of conditions intimately related with development of the pulmonary edema.

\section{METHOD}

Using the mongrel dogs weighing 10 to $25 \mathrm{Kg}$., under the anesthesia with sodium hexabarbiturate, a polyethylen tube was introduced directly into the right duct after it was visualized by Evans blue ${ }^{14)}$ given in the thoracic cavity above 20 to $30 \mathrm{~min}$. prior to surgical opening of the junctional area of the jugular and subclavian vein in the right side of the neck. Lymphatic fluid passing through the polyethylen tube introduced was measured every $5 \mathrm{~min}$.

Lymphatic fluid obtained, though not every sample, was presented for determining the total protein and its fraction.

The right heart bypass technique was applied to all the experimental dogs in order to keep the pulmonary blood flow constant. The dead space of apparatus was filled with $500 \mathrm{ml}$. of blood and an additional $100 \mathrm{ml}$. of Ringer's solution.

The left atrium was canulated by a catheter to drain the excessive amount of blood retained enough to rise the intraatrial pressure, keeping it within the normal limits.

The flow rate used for the pulmonary circuit ranged between 50 and $60 \mathrm{ml}$. per min. A respirator was connected with a tube introduced into the trachea, and respiration rate was adjusted in the range of 12 to 15 per min. under the intermittent positive pressure of 10 to $15 \mathrm{~mm}$. $\mathrm{Hg}$.

\section{RESUlts}

1) Effect of epinephrine on the lymph flow of the lung:

After the experimental animal was set as stated above, lymphatic flow had begun to collect every $5 \mathrm{~min}$. When it was confirmed that the $5 \mathrm{~min}$. lymph sample had reached a constant level in several samples, epinephrine was given in the pulmonary arterial stem at the rate of $5 \mu \mathrm{g}$. per $\mathrm{Kg}$. of body weight per min. As shown in the Fig. 1, the arterial blood pressure rose remarkably with the simultaneous reduction of the cardiac rate and the change of pulmonary arterial pressure was insignificant though occasional slight elevation was seen in other cases.

The left atrial pressure remained unchanged. The lymphatic flow in 


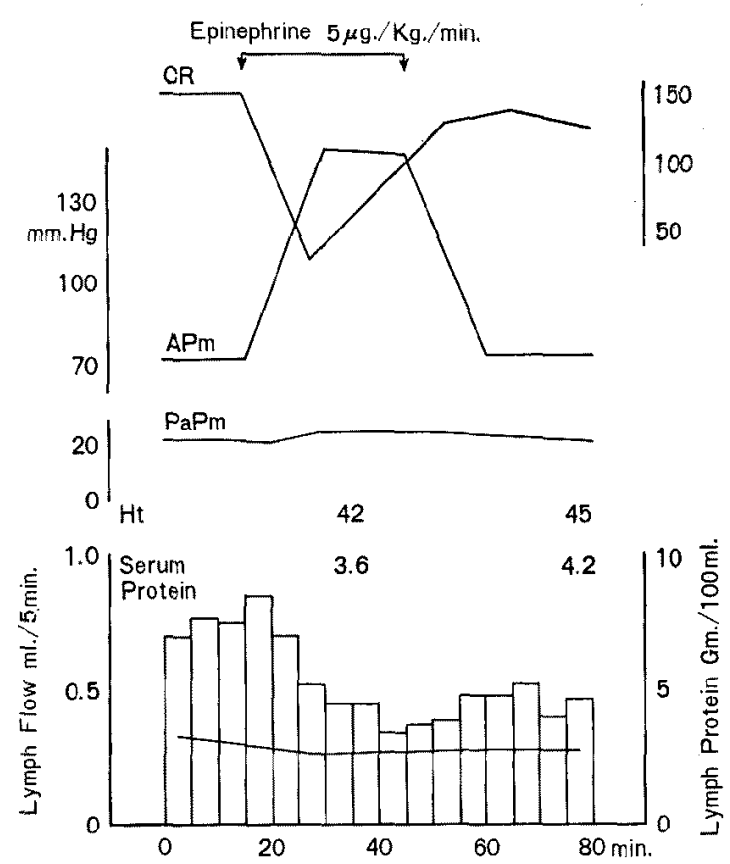

Fig. 1. Pulmonary lymph flow during epinephine infusion $(5 \mu \mathrm{g} . / \mathrm{Kg}$. min.) under constant pulmonary circulation and respiration.

each $5 \mathrm{~min}$. amounted to 0.69 to $0.76 \mathrm{ml}^{14)}$ before the additional infusion of epinephrine. The lymphatic flow was increased slightly just after epinephrine had been added to the infusate and diminished definitely from $0.84 \mathrm{ml}$. to $0.36 \mathrm{ml}$. during the period of the additional infusion of epinephrine and then resumed the gradual increase. A pattern of the initial increase with subsequent reduction was the case.

2) Effect of vagotmy on the epinephrine induced variable lymphatic flow :

Before initiating an infusion with epinephrine, bilateral vagotomies were performed on 3 dogs whose respiration was put under the control as stated previously. As it is shown in Fig. 2, the lymphatic flow measured was $0.38 \mathrm{ml}$. in the first sample (in other two $0.31 \mathrm{ml}$. and $0.27 \mathrm{ml}$. respectively) with a minimal prevagotomy variation. The lymphatic flow has been maintained to approximately equivalent in the subsequent several samples and then increased up to the level of about $0.5 \mathrm{ml}$., which was kept fairly constant even after the epinephrine administration. In other cases, the level of lymphatic flow after administration of epinephrine to the vagotomized animals demonstrated definite increase of the hemodynamic changes such as the arterial blood pressure, the pulmonary arterial pressure ; the left atrial pressure were basically 


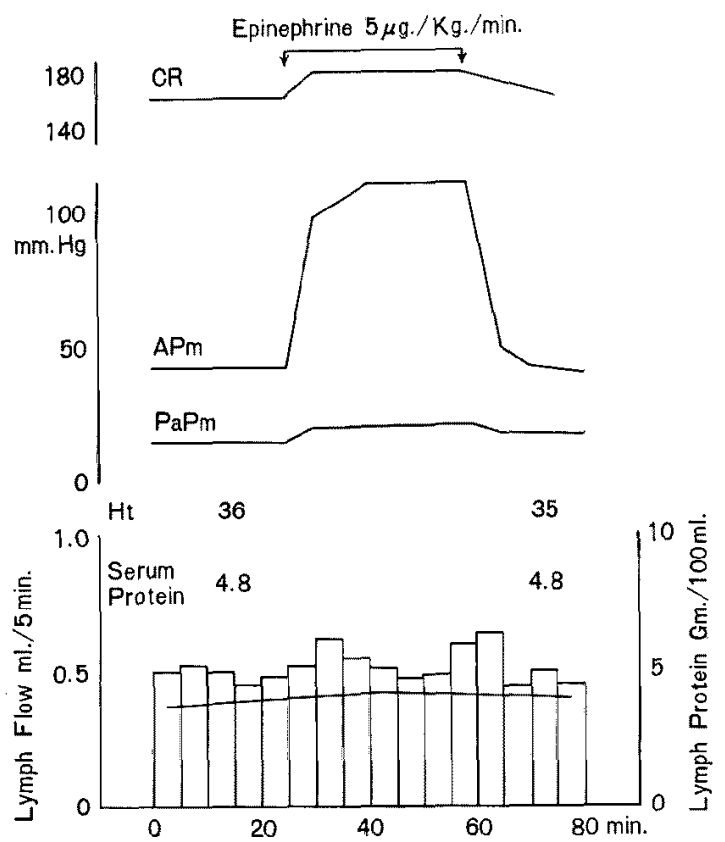

Fig. 2. Pulmonary lymph flow during epinephrine infusion $(5 \mu \mathrm{g} . / \mathrm{Kg} . /$ min.) in vagotomized dog under constant pulmonary circulation and respiration.

identical with those seen in the normal dogs with the only exception of positive chronotropic effect.

3) Effect of reserpine on the epinephrine induced variations of the lymphatic flow:

One of the experimental dogs was treated with reserpine $0.25 \mathrm{mg}$. per $\mathrm{Kg}$. of body weight for 3 days before measurement of the lymphatic flow. The mean arterial blood pressure was $50 \mathrm{~mm} . \mathrm{Hg}$ and the cardiac rate 102 per min., both of which were comparatively of low value compared with those of the untreated. When epinephrine was administered, the arterial blood pressure rose remarkably up to $128 \mathrm{~mm} . \mathrm{Hg}$ and cardiac rate also increased up to the level of 150 per min. with a transient bradycardia. The lymphatic flow maintained at the level of about $0.6 \mathrm{ml}$. per $5 \mathrm{~min}$. but marked increase up to $1.0 \mathrm{ml}$. or more was shown.

4) Effect of anoxia on the lymphatic flow:

In order to induce anoxia, a long tracheal cannula was employed aiming at enlarged dead space of the air passage. Fig. 4 shows oxygen saturation of the animal subjected to the tracheal cannulation which was shown to fall from $96.6 \%$ down to $67.3 \%$ in $35 \mathrm{~min}$. The fall of oxygen saturation depends on the size and the length of the cannula inserted and may also vary individually. 


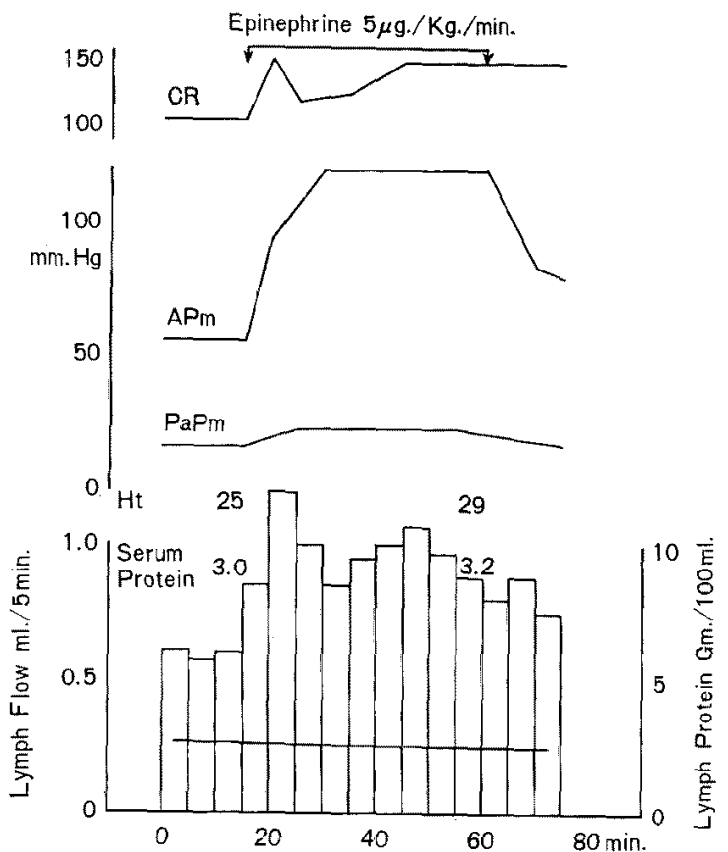

Fig. 3. Pulmonary lymph flow during epinephrine infusion $(5 \mu \mathrm{g} / \mathrm{Kg}$. min.) in reserpinized dog under constant pulmonary circulation and respiration.

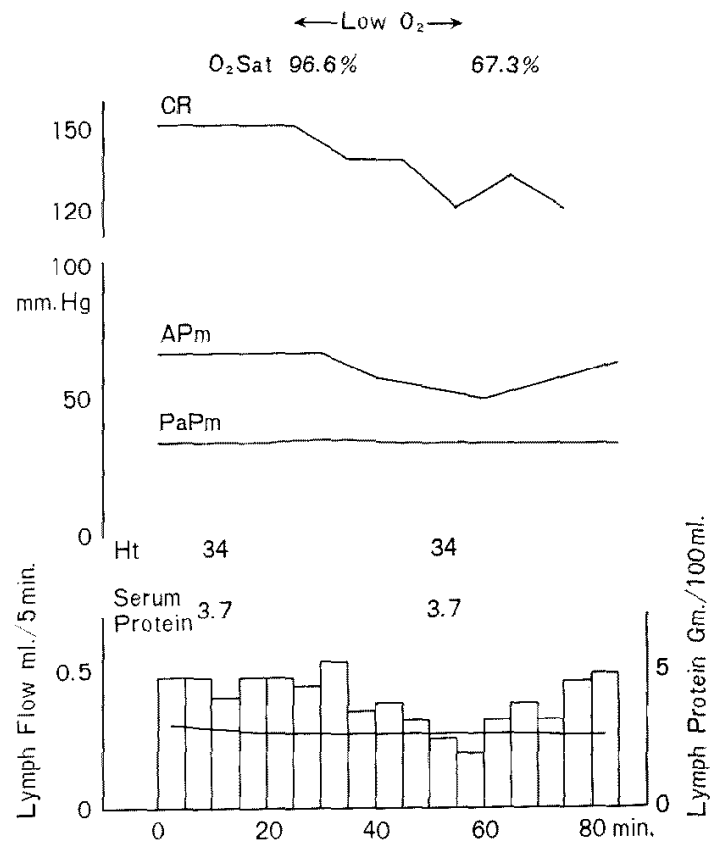

Fig. 4. Pulmonary lymph flow in induced anoxia under a constant pulmonary circulation and respiration. 
Oxygen saturation has fallen remarkably to the least figure of $67.3 \%$, as far as the present experiments go. In Fig. 4, the arterial blood pressure has shown no remarkable change during the early half period of induced anoxia but tends to fall in the later half until reaching to the considerable low level below $50 \mathrm{~mm} . \mathrm{Hg}$. The cardiac rate has shown to run fairly parallel with the change of the arterial blood pressure. The pulmonary arterial pressure remained the same in this case but has been found to rise slightly in other cases.

The lymphatic flow ranged from 0.48 to $0.40 \mathrm{ml}$. per $5 \mathrm{~min}$. before insertion of the tracheal cannula, then increased up to the level of $0.53 \mathrm{ml}$. for the early several min. of the induced anoxic period which tends to decrease fairly rapidly to the level of around $0.2 \mathrm{ml}$. per $5 \mathrm{~min}$. After discontinuing the anoxic procedure, the lymphatic flow had restored gradually toward the control level in about $20 \mathrm{~min}$. The pattern of the lymphatic flow obtained by the anoxic state is similar to that of epinephrine injection.

5) Effect of reserpine on the anoxia induced variation of the lymphatic flow:

Two dogs reserpinized in the same manner as previously described have been used for measurement of the lymphatic flow under the anoxic state. In one example, the hemodynamic changes as well as variations of the lymphatic

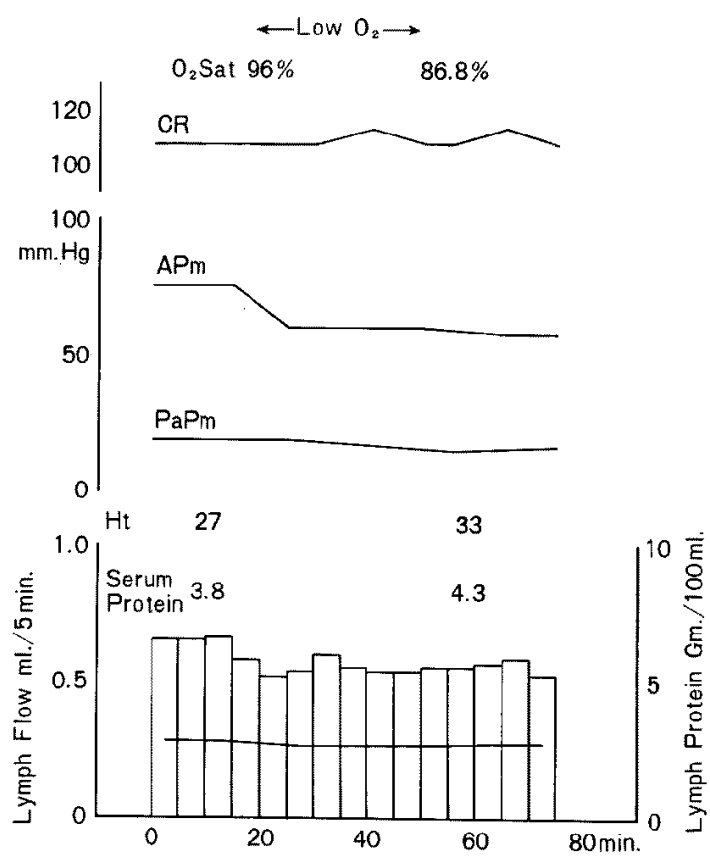

Fig. 5. Pulmonary lymph flow in induced anoxia in reserpinized dog under a constant pulmonary circulation and respiration. 
flow were shown in Fig. 5.

The hemodynamic changes have not been remarkable, but rater suppressed. As to the lymphatic flow, the preanoxic level of $0.65 \mathrm{ml}$. has been found to go down to the level usually below $0.55 \mathrm{ml}$. during the anoxic period.

Likewise in case of epinephrine, combination of the early increase, though slight, and the subsequent reduction assumes the same pattern obtained in this case.

\section{Comment}

As it is hardly possible to measure capillary permeability of lung in situ, the direct determination of the lymphatic flow from the right duct has been tried basing on the view that the lung lymph flow can serve as an index of permeability of the lung capillary, because, as stated previously, increased lung lymph can be attributed to an excessive transudation of the lung capillary beyond an attractive force of the effective osmotic pressure to draw fluid inward. This is justifiably accepted, because the electronmicroscopic studies have revealed that filtrate of the lung capillary accumulate in the interstitial tissue ${ }^{15}$ with resultant alveolar wall edema filling the alveolar space. ${ }^{16)}$ Filtrate of the lung capillary which is ready to accumulate in the interstitial tissue would contribute to heighten the lymphatic flow until increased interstitial fluid reached the level beyond capacity of the lymphatic drainage.

It was found that the pulmonary lymphatic flow had increased in proportion to the rate of pulmonary blood flow and the level of the left atrial pressure, as will be reported elsewhere. ${ }^{17)}$ These 2 hemodynamic components should be kept constantly for evaluating permeability of the lung capillary by serving the lymphatic flow as an index. It is important, therefore, to note in these experiments that the right heart by pass technique has been applied combined with the left atrial cannulation which is tied with the blood reservoir, resulting in maintaining the hemodynamic constancy. Respiration has been kept unchanged. Under these circumstances the lymphatic flow would reflect permeability variations of the lung capillary.

Epinephrine, when infused into the pulmonary circuit under the above conditions, has caused the transient but early increased lymphatic flow with subsequent reduction which has been accompanied by rise in the arterial blood pressure which in turn decreased the cardiac rate. It seems highly probable that the variations of the lymphatic flow due to epinephrine are associated, related with the sympathetic effect attended by vagal discharge.

If so, when the vagal discharge is suppressed or blocked, sympathetic effect would be unmasked. Thus, vagotomy has been attempted, and it has 
been demonstrated that in the vagotomized dogs increased lymphatic flow upon administration of epinephrine has continued throughout on infusion period or the subsequent reduction of the lymph flow has remarkably suppressed. It is reasonably assumed that the eventual decrease of the lymph flow has resulted from the vagal discharge elicited through the baroreceptor reflex mechanism. In the reserpinized dog, considerably increased lymphatic flow signifies that the sympathetic discharge is acting as a potent accelerator of the lung capillary permeability.

It has been noted that anoxia can exert an influence directly on the capillary membrane, resulting in increased transudation. $\left.{ }^{6},{ }^{7}\right)$ Under the circumstances of the stationary hemodynamic state of the pulmonary circuit except some changes, if any, induced by anoxia, it would be justifiably assumed that the variable lymphatic flow reflect the direct effect of anoxia on the lung capillary. Anoxic state has caused a similar pattern of the lymphatic flow to that resulting from epinephrine infusion, that is to say, the initial increase with the subsequent reduction, though the degree of lymphatic flow varies independent of the percentage of oxygen saturation. In fact, hypoxia has been found to be in the range from $61.8 \%$ to $17.0 \%$ applying the technique specially devised for lowering oxygen saturation. The initial phase of increased lymphatic flow is not so pronounced that it is not likely that so far as the present experiments are concerned the accelerating effect of anoxia on transudation of the lung capillary can be evaluated similarly as in the previous reports. ${ }^{18)}$ Although the hemodynamic changes induced have been negligible, it seems probable that the pattern of the lymphatic flow so obtained is related to the increased sympathetic tone, in view of the fact that anoxia has been proved to be associated with increased secretion of catecholamine. As has been attempted in case of epinephrine, the reserpinized dogs have been employed in order to learn whether the pattern of the lymphatic flow is attributable to the increased sympathetic tone possibly induced by anoxia. As the induced level of oxygen saturation in the reserpinized dog has not been significantly low, it does not seem, that the lymphatic flow has been influenced remarkably. It has been found that no profound increase was observed even in the initial phase of anoxia, however, no subsequent reduction had occurred. Although it is difficult to state that the anoxic effect on the lymphatic flow is entirely due to an increased sympathetic activity as shown with the sympathomimetic agent, it would be safe to interpret that the sympathetic activity is involved in the anoxic effect at least in part.

It is well known that epinephrine can produce pulmonary edema in the animals as a result of circulatory embarrassment of the pulmonary circuit. Speaking of the circulatory embarrassment, increased venous return and in- 
competence of the left ventricle are responsible for development of pulmonary edema. ${ }^{19), 20)}$ Besides these hemodynamic changes, it has been shown that epinephrine can increase transudation of the pulmonary capillary, though it is suppressed by vagal activity elicited through baroreceptor reflex mechanism. Whether stimulation of the sympathetic nerve innervating the lung can cause transudation of the lung capillary to increase remain to be investigated. It is likely, however, that epinephrine may affect the capillary membrane of the lung to make increase permeability. On the other hand, the fact that vagotomy is invariably followed by increased lymphatic flow and suppresses the subsequent reduction of the lymphatic flow in the later stage of epinephrine infusion, that is, the vagal effect, indicates that vagal discharge exerts suppressive influence on transudation of the lung capillary. In anoxic state the suppressive effect of vagal activity on capillary transudation could be well demonstrated by the vagotomized dog, though sympathetic acceleration could hardly be demonstrated unequivocally.

\section{Summary AND Concluston}

(1) With the dog maintaining the pulmonary circulation stable, permeability of the lung capillary has been observed by an indirect method of determining the lymphatic flow of the lung.

(2) In order to keep the pulmonary circulation stable the right heart by pass technique has been employed with the left atrial cannulation for draining the excessive blood to prevent the intraatrial pressure from rising.

(3) Epinephrine infusion was invariably followed by the initial increase and the subsequent reduction in the lymphatic flow of the lung.

(4) In the vagotomized dog, the lymphatic flow was found to increase and the subsequent decrease of the later stage of epinephrine infusion was suppressed.

(5) In the reserpinized dog, the lymphatic flow was increased remarkably by epinephrine infusion.

(6) In anoxic state, the lymphatic flow varied with the similar pattern to that of epinephrine, and was prevented from reduction occurring in the later stage by vagotomy.

(7) It seems highly probable that catecholamine and probably sympathetic stimulation exerts an accelerating effect on transudation of the lung capillary and that vagal discharge may act as a suppressor. 


\section{REFERENCES}

1. Ruch, T. G. and Fulton, J. F.: Medical Physiology and Biophysics. Saunders Company, Philadelphia and London, p. 758, 1960.

2. Hendley, E. D. and Schiller, A. A.: Am. J. Physiol. 180: 378, 1955.

3. Inchley, O.: J. Physiol. 61: 282, 1926.

4. Ueno, M.: Jap. J. Int. Med. 49: 107, 1960.

5. Maurer, F. W.: Am. J. Physiol. 133: 170, 1941.

6. Landis, E. M.: Am. J. Physiol. 83: 528, 1928.

7. Drinker, C. K.: Pulmonary edema and inflammation. Harvard Univ. Press, Cambridge, 1945.

8. Landis, E. M.: Physiol. Rev. 14: 404, 1934.

9. Campbell, G. S., Maddy, F. J., Adams, W. L., and Visscher, M. B.: Am. J. Physiol. 158: 96, 1949.

10. Jarisch, A., Richter, H., and Thoma, H.: Klin. Wschr. 18: 1440, 1939.

11. Gamble, J. E. and Patton, H. D.: Science 113: 626, 1951.

12. Ueba, Y.: Jap. Circulat. J. 24: 648, 1960.

13. Sarnoff, S. J. and Berglund, E.: Am. J. Physiol, 170: 588, 1952.

14. Akase, M.: Jap. Circulat. J. 24: 1459. 1960.

15. Yoshida, M.: Bull. Kobe Med. Coll. 18: 1367, 1960.

16. Staub, N. C.: This Symposium.

17. Oka, K. and Maeda, T.: To be published.

18. Visscher, M. B., Haddy, F.J., and Stephens, G.: Pharmacol. Rev. 8: 389, 1956.

19. Tomomatsu, T. and Yorifuji, S.: Jap. Circulat. J. 25: 503, 1961.

20. Tomomatsu, T., Inoh, T., Matsumoto, E., Koterazawa, M., Maeda, T., and Yoshitake, K.: Jap. Circulat. J. 29: 353, 1965. 